\title{
Actitudes matemáticas y rendimiento escolar
}

\section{Nieves Quiles}

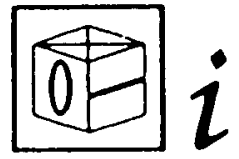

La bipótesis aptitudinal, se invoque o no el innatismo para sostenerle, es la predilecta para explicar el fracaso de los alumnos en matemáticas. Otra muy distinta aunque complementaria sería la adecuación de la instrucción y la aptitud educativo-matemática del docente. Entre ambas, cabría situar las actitudes de los actores: alumnos y profesores. En esta investigación se analiza $y$ confirma la relación entre rendimiento y actitudes.

\section{INTRODUCCION}

Las destrezas intelectuales que los estudiantes adquieren en la escuela no se restringen a los contenidos de las asignaturas de su currículum. También aprenden normas de irteracción, modos de comunicación, concepciones sobre el mundo, actitudes hacia lo que les rodea, etc. De este vago conjunto de creencias que se desarrollan en el ámbito escolar, una de las que ha adquirido mayor importancia por sus repercusiones en el rendimiento académico es la actitud positiva o negativa hacia cada asignatura. Especialmente hacia las matemáticas, ya que ésta parece ser la materia que concentra más sentimientos contrapuestos.

Desde sus orígenes, la Psicología Social se ha preocupado por el estudio de las actitudes, interés que comparte cuanto menos con la Sociología y con la Educación. Pero a pesar de la gran cantidad de investigación generada a lo largo de estos años su análisis sigue plagado de equívocos y dificultades, especialmente a la hora de tratar la relación actitud y conducta. Presentaré de manera suscinta las principales tesis básicas que se mantienen desde la Psicología Social de la Educación acerca de esta conexión.

En primer lugar, las correlaciones halladas entre las variables actitud y conducta aunque significativas son relativamente bajas, dándose esta circunstancia en todos los niveles escolares y diferente origen nacional (Do Carmo de Avila y Gillet, 1970; Kulharni y Naidu, 1970). Más aún, según los resultados encontrados por Spickerman (1970) la actitud matemática está tan relacionada con 
la nota real como con la nota deseada, y los resultados obtenidos por Jackson (1974) apuntan a que la conexión actitud-rendimiento en matemáticas sólo se da cuando las actitudes son extremas, esto es, cuando son muy positivas o muy negativas.

En segundo lugar, se ha advertido que las actitudes matemáticas de los padres y profesores explican buena parte de la varianza de las actitudes de los alumnos hacia la asignatura (Banks, 1964). En contraste, otros autores apuntan que es la actitud del profesor y su eficacia en la enseñanza lo que constituye el determinante fundamental de la actitud y el rendimiento del estudiante (Aiken y Dreger, 1961; Aiken, 1972; Berstein, 1964). Los partidos de esta línea subrayan la necesidad de intervenir sobre los futuros profesores de matemáticas por su potencial influencia sobre los alumnos. Fennema (1980), liquida el debate señalando que los efectos de las actitudes del profesor y su conducta sobre el rendimiento del alumno varían de manera importante de un profesor a otro y de un estudiante a otro.

En tercer lugar, los resultados acerca de la influencia de las actitudes paternas no son más satisfactorios que los anteriores. Aún se sigue desconociendo la amplitud de la interacción de las actitudes de las madres y de los padres con el rendimiento de sus hijos e hijas. La naturaleza poco clara de esta relación ha generado un número destacado de trabajos que, en general, hablan de una correlación positiva. Lo que no está enteramente dilucidado es el alcance de la interacción. De acuerdo con Poffenberger y Norton (1959), uno de los medios con que cuentan los padres para afectar las actitudes y rendimiento de sus hijos son sus propias actitudes. También la opinión de los padres acerca de la importancia y necesidad de las matemáticas en la vida moderna y la percepción de las actitudes paternas por parte del alumno están significativamente relacionadas con la actitud y el rendimiento del estudiante (Alpert et al., 1963; Fennema y Sherman, 1977). Por su parte, Burbank (1968) establece un vínculo diferente de las actitudes del padre y de las actitudes de la madre. Mientras las primeras se relacionan positivamente con el rendimiento, las segundas inciden de modo especial en las actitudes propiamente dichas de los hijos.

De manera genérica, el punto de partida del presente trabajo es la consideración de las actitudes como predisposiciones aprendidas que nos llevan a actuar de una forma determinada ante personas y situaciones. Las experiencias escolares y las influencias familiares constituyen elementos importantes en la adquisición y cambio de las actitudes de los estudiantes. El propósito de este estudio es profundizar en la relación actitud hacia las matemáticas y rendimiento en dicha asignatura observando la actitud del alumno, su profesor y sus padres en el mismo contexto de investigación. En mi opinión, las actitudes hacia las matemáticas influyen necesariamente, y sin menosprecio de otras variables, en el tiempo y el esfuerzo dedicados a trabajar cuestiones relativas a esa asignatura y esto, a su vez, repercute en el rendimiento y la nota obtenida. De todos es sabido que una actitud positiva facilita el aprendizaje mientras que una actitud negativa lo dificulta. No es extraño, por tanto, que desde el punto de vista de la Educación en general y de las Didácticas en particular el concepto esté revestido de gran importancia. En el primer caso tenemos ejemplos palpables de esta preocupación por las actitudes - de alumnos, profesores y familiarescuando nos centramos en la política de integración de deficientes físicos o en el terreno de los alumnos y profesores afectados de SIDA. En el segundo caso las actitudes como predisposiciones para el aprendizaje adquieren un significa- 
do especial. De manera que, independientemente del objeto de la actitud (asignatura, profesor, escuela...) sabemos que convienen actitudes positivas (Gairín, 1987). Aun así, desconocemos en qué medida el contexto social que representa la actitud de padres y profesores afecta al rendimiento del niño.

\section{METODO}

\section{Sujetos}

Para realizar este estudio seleccionamos una muestra de tres poblaciones distintas: a) 600 alumnos de $5^{\circ}$ de EGB procedentes de 15 colegios públicos de la isla de Tenerife, con edades comprendidas entre 9 y 15 años. De éstos, el $55 \%$ eran varones y el $46 \%$ residían en un hábitat urbano. b) Sus padres, en total 300 , cuyas edades oscilaban entre los 26 y los 70 años. c) Sus profesores, 24 distribuidos uniformemente según el sexo y la ubicación del centro escolar - rural vs. urbano-. La edad media de los maestros fue de 36,9 años. La distribución según la especialidad seguida durante la carrera fue la siguiente: Humanas, 7; Naturales, 6; Lenguaje, 2; Preescolar, 2, y Ninguna, 7. Los profesores impartían la casi totalidad de las asignaturas correspondientes a 5. curso de EGB.

\section{Instrumentos}

Empleamos tres escalas de actitud matemática tipo Likert, elaboradas para este estudio. Parte de los enunciados que figuran en las escalas fueron de elaboración propia y parte fueron extraídos de las escalas $\mathrm{E}$ (Enjoyment of mathematics) y V (Value of mathemantics) de Aiken (1972), y de la Escala de Dutton (1954). La más extensa era la correspondiente a los alumnos, que constaba de 35 ítems. Las dos restantes tenían 28. Estas escalas quedaron reducidas a 23 ítems después de conocer la correlación ítem-test (ver anexos $\mathrm{n}^{\circ} 1 \mathrm{y} \mathrm{n} \mathrm{n}^{\circ} 2$ ).

Todas ellas recogían proposiciones favorables y desfavorables a las matemáticas, que los sujetos debían contestar atendiendo a una escala de 5 puntos, cuya codificación daba el valor máximo a la respuesta más favorable hacia las matemáticas.

La fiabilidad de las tres escalas se determinó mediante el procedimiento ideado por Cronbach para averiguar la consistencia interna de un test. Las puntuaciones alpha obtenidas fueron altas en los tres casos $(0.89$ para la escala de niños y padres; 0.92 para la de maestros).

\section{Procedimiento}

Excepto en el caso de los padres que optamos por enviarles las pruebas por medio de sus hijos, y bajo el control del profesor, la recogida de datos se llevó a cabo en el centro escolar. Colectivamente con los niños e individualmente con los profesores. En el mes de junio se volvió a los colegios y se recogieron las notas finales de los alumnos (circunstancia que sólo conocía, previamente, el director del centro). 


\section{8}

\section{RESULTADOS}

Los resultados se presentan en dos bloques diferentes. En el primero analizamos la factorialización de las distintas escalas, y en el segundo exponemos los datos que constituyen el interés fundamental de esta investigación.

\section{Análisis de las escalas de actitud hacia las matemáticas}

En el análisis factorial de la matriz de correlación de los ítems de la escala de padres y de la escala de alumnos (no se realizó con la escala de maestro por el tamaño reducido de la muestra), se obtuvo una solución de tres factores en cada escala con valores propios iguales o superiores a 1 . Ambas soluciones factoriales fueron sometidas a una rotación ortogonal por el método varimax, para lograr una descomposición de variables parecida a la esperada teóricamente, y obtenida en estudios realizados en el ámbito anglosajón.

Los factores obtenidos serán comentados a continuación, y se acompañan de una clave que permite identificar las cuestiones de cada factor en las escalas presentadas en el anexo.

\section{Escala de actitud matemática para los alumnos de EGB}

Tres fueron los factores derivados de esta escala de actitud. El primero de ellos que denominamos agrado por las matemáticas $(A G R)$ obtuvo un valor propio de 7.6 y explica un porcentaje de varianza del $33 \%$. Los ítems con saturaciones superiores a 0.30 se refieren básicamente al divertimiento o goce que supone la realización de actividades relacionadas con las matemáticas o con el estudio de esa asignatura (ítem $16, \mathrm{r}=0.80$ ).

El segundo factor alude al temor a las matemáticas (TE). No obstante, la naturaleza de los ítems que correlacionan con este factor sugieren fórmulas distintas para expresar ese miedo hacia las matemáticas. Reflejan sentimientos negativos (incluso de odio) hacia la asignatura, tal y como se observa en estas cuestiones: «Las matemáticas me hacen sentir incómodo y nervioso» $(\mathrm{r}=0.70)$; «Nunca me han gustado las matemáticas y es la asignatura que más temo» ( $\mathrm{r}=0.74)$; «Generalmente, odio las matemáticas y evito usarlas siempre que puedo» $(r=0.65)$. El valor propio obtenido para este factor fue de 1.8 , que explica el $7,9 \%$ de la varianza.

La índole de las variables reunidas en el tercer factor indican el valor o utilidad de las matemáticas, no sólo en el currículum escolar, sino en la sociedad, como un instrumento de progreso y de valía intelectual. Por ello le etiquetamos como Utilidad de las matemáticas (UTI). Con un valor propio de 0.9 , y un porcentaje de varianza explicado de 3.9 , los ítems de este factor señalan no sólo la necesidad $(r=0.60)$ sino la obligación de todo el mundo de estudiar matemáticas $(r=0.57)$.

\section{Escala de actitud matemática para adultos}

Los tres factores obtenidos en la escala aplicada a los padres de los alumnos de 5 . de EGB fueron Desagrado y temor a las matemáticas (F1),. Agrado y utilidad de las matemáticas (F2) y Desvalorización de las matemáticas (F3). Los dos primeros factores presentan un mayor valor propio, el más poderoso lo constituye el de temor a las matemáticas (TE) - valor propio de 6.8- mientras el factor 
Agrado y utilidad de las matemáticas (AGR y UTI) obtuvo un valor propio de 1.9. El porcentaje de varianza que explicaba el primer factor fue de $29,5 \%$, mientras el segundo explicaba un $8,2 \%$. Los ítems representativos de uno y otro factor son semejantes a los detectados en la escala de los niños.

El tercer factor Desvalorización de las matemáticas (DES), denominado así porque los ítems más saturados en el factor buscan descalificar las matemáticas, que no tienen ningún valor $(r=0.66)$, ni son importantes $(r=0.54)$, presenta un valor propio de 1.3 y explica el $5,5 \%$ de la varianza.

\section{Las actitudes y el rendimiento en matemáticas}

Una vez determinadas las puntuaciones de los alumnos y de los padres en cada factor de la escala, así como en la escala global, se procedió a determinar las correlaciones entre esas puntuaciones y la nota final obtenida por el alumno en matemáticas. Este cálculo se completó con el estudio pormenorizado de la función predictora de cada variable mediante el análisis de regresión. A continuación presentamos los resultados para cada una de las muestras analizadas: padres, profesores y alumnos.

\section{Las actitudes de los padres}

La correlación entre la actitud global, los tres factores de la escala y las notas del niño en matemáticas (rendimiento) se presentan en el Tabla I.

TABLA I

Correlación de Pearson entre "Actitud paterna» bacia las matemáticas (escala global y cada factor) y «Nota del niño en matemáticas»

\begin{tabular}{|l|c|c|c|c|}
\hline & $\begin{array}{c}\text { Actitud } \\
\text { global }\end{array}$ & $\begin{array}{c}\text { Desagrado } \\
\text { matem. }\end{array}$ & $\begin{array}{c}\text { Agrado } \\
\text { matem. }\end{array}$ & $\begin{array}{c}\text { Desvaloriz. } \\
\text { matem. }\end{array}$ \\
\hline NOTA MATEM. & $0.15^{*}$ & $0.14^{*}$ & 0.10 & $0.12^{*}$ \\
\hline
\end{tabular}

$* \mathrm{p} \leqslant=.05$

Como se observa, en tres de las cuatro comparaciones se alcanza la significación. Cierta es que existe una relación pequeña, aunque no despreciable, entre las distintas variables actitudinales del padre y la nota lograda por el niño. Es, curiosamente, el factor agrado por las matemáticas el que roza la significación sin conseguirla, mientras que los dos factores negativos sí la alcanzan. Este hecho se repite de nuevo en la correlación de la actitud y el rendimiento del alumno (Tabla II). De modo que, de forma general, y a la luz de las correlaciones obtenidas, el rendimiento del niño parece «tener algo que ver» con las actitudes de los padres hacia las matemáticas.

Con el fin de estudiar más minuciosamente este detalle, ejecutamos un análisis de regresión múltiple empleando como varibles predictoras todas las puntuaciones paternas y como variable criterio, las notas. El porcentaje de varianza explicado no sobrepasa el $2,5 \%\left(R^{2}=0.024 ; F<(4,308)=1.90\right)$ lo que, en contra de lo esperado, advierte del reducido papel que juegan las actitudes de los padres. Esto no hace más que poner en evidencia la natura- 
leza poco clara de la relación entre ambos tipos de variable. A pesar de la gran cantidad de investigación generada sobre el tema seguimos desconociendo la intensidad de la misma.

\section{Las actitudes de los profesores}

Si los resultados relativos a los padres resultaron decepcionantes, más aún lo fueron los obtenidos por los profesores. Tal y como apuntábamos en la introducción desde el punto de vista de distintos autores los profesores se consideran como causantes directos de las preferencias o rechazos de sus alumnos por las asignaturas que imparten. Esto hizo que «a priori» esperáramos una mayor determinación de las actitudes del maestro; sin embargo, los resultados de nuestros análisis fueron concluyentes al señalar una correlación mínima entre las actitudes del profesor y la medida del rendimiento del alumno $(r=0.013)$ el análisis de regresión es igualmente despreciable $\left(\mathrm{R}^{2}=0.00017\right)$.

\section{Las actitudes del alumno}

Una vez analizada la relación entre la actitud de «los otros» - en este caso concreto traducido en padres y profesores- y la nota final obtenida por el niño en matemáticas, pasamos a tratar el papel que las propias actitudes del estudiante desempeñan en su rendimiento en la citada asignatura. $\mathrm{Al}$ igual que en los casos anteriores vamos a tener en cuenta la puntuación media obtenida en la escala global y la puntuación media de cada factor.

El coeficiente de correlación de Pearson indicó la existencia de relación entre las cuatro variables predictoras (actitud global y los tres factores de la escala) y la nota final en matemáticas. Estas correlaciones no son excesivamente altas, puesto que oscilan entre $r=0.18$ y $r=0.27$, pero se mueven alrededor de los valores obtenidos por otros estudios (véase Tabla II).

TABLA II

Correlación de Pearson entre «Actitud del niño» bacia las matemáticas (escala global y cada factor) y «Nota del niño en matemáticas»

\begin{tabular}{|l|c|c|c|c|}
\hline & $\begin{array}{c}\text { Actitud } \\
\text { global }\end{array}$ & $\begin{array}{c}\text { Agrado } \\
\text { matem. }\end{array}$ & $\begin{array}{c}\text { Temor } \\
\text { matem. }\end{array}$ & $\begin{array}{c}\text { Valor } \\
\text { matem. }\end{array}$ \\
\hline NOTA MATEM. & $0.27^{* *}$ & $0.18^{*}$ & $0.27^{* *}$ & $0.21^{*}$ \\
\hline
\end{tabular}

$* \mathrm{P} \leqslant=0.05$

${ }^{*} \mathrm{p} \Pi \leqslant=0.01$

Como podemos ver, si atendemos a los distintos factores, es el temor a las matemáticas - más que el agrado por las mismas - el que presenta un valor mayor. Mientras, el placer que supone el trabajar la asignatura se sitúa en el lugar más bajo de las tres correlaciones. Por su parte, la importancia que se otorga a la asignatura obtiene el valor intermedio. Por tanto, la relación más estrecha con la nota final se da con el miedo o el desagrado 
que experimentan los alumnos hacia la materia. La intensidad de la correspondencia es idéntica en el caso de la actitud global.

Respecto al análisis de regresión, éste nos indicó que las cuatro variables predictoras utilizadas explicaban un $9,8 \%$ de la varianza en la nota final del niño. De este porcentaje total el dato más destacable es que la varianza común de las distintas variables — más que la aportación particular de alguna de ellas- es la que explica un mayor porcentaje de varianza en la nota final.

\section{TABLA III}

Valores obtenidos en el análisis de regresión múltiple con las variables actitudinales del niño y la nota final como variable criterio

\begin{tabular}{|l|l|l|l|}
\hline $\mathrm{R}^{2}$ & $=0.09812$ & $\mathrm{gl}=4$ & $\mathrm{~F}=14.52337$ \\
$\varphi \mathrm{r}^{2}$ ACTITUD GLOBAL & $=0.01541$ & $\mathrm{gl}=534$ & \\
$\varphi \mathrm{r}^{2}$ AGRADO MATEMATICAS & $=0.01416$ & $\mathrm{gl}=534$ & \\
$\sum \varphi \mathrm{r}^{2}{ }_{\mathrm{i}}$ (VARIANZA UNICA) & $=0.02958$ & & \\
$\mathrm{R}^{2}-\Sigma \varphi \mathrm{r}^{2}{ }_{\mathrm{i}}$ (VARIANZA COMUN) & $=0.06854$ & & \\
\hline
\end{tabular}

\section{DISCUSION}

Tal y como se recoge en el apartado dedicado a los resultados, las mediciones que se llevaron a cabo nos permitieron, en un primer análisis, conocer la actitud global de los profesores hacia la asignatura de matemáticas así como la de padres y alumnos. En un segundo momento tratamos de observar la relación entre las actitudes de cada uno de los tres grupos (padres, profesores y alumnos) y la nota final de los estudiantes en la asignatura de matemáticas. A lo largo de esta discusión presentaré argumentos que ayuden a clarificar los resultados alcanzados, así como algunas de las posibilidades que, desde mi punto de vista, se abren para los docentes y la práctica didáctica.

La actitud hacia las matemáticas se define como la tendencia por parte de los individuos a responder, positiva o negativamente, a la asignatura. La importancia de desarrollar actitudes positivas hacia una materia determinada se considera como un medio para preparar al estudiante a usar las habilidades y conocimientos adquiridos, así como para acercarle al estudio de dicha materia (los sentimientos negativos llevan a su rechazo).

Distintas revisiones sobre el tema destacan a las actitudes paternas como una de las variables que afectan de forma relevante no sólo a la elaboración de actitudes positivas hacia las matemáticas sino también al nivel de rendimiento logrado por el alumno. Si atendemos a la incidencia de las actitudes paternas sobre el nivel de rendimiento logrado por el niño, nuestros resultados van en la línea general de las distintas investigaciones. Es decir, encontramos relaciones significativas y positivas (pero bajas) entre las variables.

No debemos olvidar, sin embargo, que la falta de acuerdo entre los distintos estudios es una constante en este terreno. Los resultados van desde una ausencia total de relación entre las variables (Aiken y Dreger, 1961) hasta relaciones más o menos altas (Wildhem y Brooks, 1980). Hecho que se 
ve facilitado por la ausencia de modelos teóricos que permitan integrar esta diversidad de resultados y conocer el verdadero alcance de las actitudes paternas en las actitudes y rendimiento de sus hijos.

Obviamente, la contribución de dichas actitudes tendría menor relevancia si no afectaran en alguna medida al rendimiento de los alumnos.

Por lo que se refiere a las actitudes del profesor, según nuestro trabajo, no parecen afectar al nivel de rendimiento del alumno, lo que, a pesar de los resultados contradictorios en este campo no deja de ser sorprendente. Sobre todo si tenemos en cuenta que un número importante de estudios sobre el tema, mantienen que la actitud del profesor hacia una asignatura particular determina, en cierta forma, la actitud y el rendimiento del alumno (Banks, 1964; Aiken, 1970). Sin embargo, el mismo Aiken (1976), en una revisión posterior, concluye que resulta difícil confirmar esta relación.

A pesar de nuestros resultados, seguimos creyendo que las actitudes matemáticas del profesor tienen algún tipo de incidencia sobre el rendimiento de sus alumnos. El hecho de que tales relaciones no estén presentes en nuestro trabajo puede deberse a su efecto «techo» producido por nuestra escala de actitud con los profesores. En efecto, el análisis más elemental de nuestros datos mostraba una actitud extremadamente favorable de los profesores hacia las matemáticas (sólo un 5,3\% reconocía una actitud moderadamente desfavorable y un $0 \%$ señalaba una actitud totalmente desfavorable). $Y$ en investigaciones anteriores (Quiles, 1986, 1988) encontramos que fue muy superior el efecto de las opiniones del profesor cuando éstas eran negativas o de rechazo que cuando eran positivas.

Igualmente, nos remitimos al hecho de que, aunque los profesores presenten actitudes positivas o favorables hacia las matemáticas, si no se traducen en conductas efectivas en el aula (forma de organizar las tareas escolares, de organizar la clase, la claridad de exposición, etc.) es posible que esta actitud pase inadvertida para los alumnos. No es sólo la actitud del profesor hacia una asignatura particular sino, además, su eficacia en la enseñanza de la misma lo que va a determinar en gran medida la actitud y el rendimiento del alumno (Bank, 1964; Aiken, 1972; Shaughnessy et al., 1983).

También nos inclinamos a pensar que las actitudes que reconocen tener los profesores hacia las matemáticas (extremadamente favorable) no se corresponden totalmente con las que en realidad se tienen. Creemos que, en este caso concreto, la deseabilidad social ha podido contaminar la respuesta, dándose, por tanto, un desfase entre sus conductas en el aula y las actitudes que teóricamente reconocen.

$\mathrm{Si}$ a todo ello unimos la circunstancia de que son los cursos de $7^{\circ}-8^{\circ}$ de EGB o los iniciales de la enseñanza media (FP, BUP) los que favorecen la influencia de las actitudes matemáticas del profesor (coinciden estos cursos con el período crítico de formación de actitudes matemáticas), la ausencia de relación presente en nuestro trabajo parece, al menos parcialmente, justificada.

De nuevo, al tratar de determinar la importancia de la actitud matemática del alumno como predictora del rendimiento, se hace patente la controversia existente en este campo. No obstante, hay mayor consistencia a la hora de situar el valor de la correlación entre ambas variables. Esta oscila entre un rango de 0.20 y 0.40 , con lo que podemos considerar que nuestros resultados se incluyen en esta generalidad al obtener correlaciones que van de 
$r=0.18$ a $r=0.27$. Mayor es también el porcentaje de varianza explicado en el rendimiento del niño.

No hay que olvidar que el rendimiento tiene carácter multidimensional, de manera que las variables actitudinales son un factor más a tener en cuenta, no el único. Pero coincidimos con Webb (1972) en señalar a la actitud matemática como uno de los predictores más importantes de su rendimiento. Dicha actitud, junto con las variables aptitudinales o las habilidades que el niño posea, explica el porcentaje de varianza más destacado en la nota final de éste (Quiles, 1986). Esta importante relación entre actitud matemática del alumno y rendimiento predomina de forma clara a lo largo de los distintos cursos académicos y para estudiantes de distintos países, tal y como señalábamos en la introducción de este trabajo. En definitiva, los resultados descritos en este artículo refuerzan la idea que implícitamente ha orientado el presente estudio: los sentimientos hacia las matemáticas de los padres, profesores y alumnos no son independientes del nivel de rendimiento logrado por el niño en la asignatura.

Respecto a las implicaciones de las actitudes en la docencia sus posibilidades son muchas y variadas. El espectro abarca desde el aprendizaje de actitudes y valores hasta el cambio de actitudes hacia colectivos diferentes y/o marginales como los deficientes, los gitanos o los afectados de SIDA, por citar sólo algunos ejemplos refrendados por la práctica cotidiana. La experiencia nos muestra de forma clara cómo las actitudes de los niños están, en muchos casos, contaminadas por las actitudes de los adultos. Igualmente, conocemos que la simple promulgación de leyes no es efectiva para lograr los objetivos propuestos si no va acompañada de un cambio de actitud de la población a la que van dirigidas. El campo de las actitudes escolares se muestra vasto y extenso y son muchos los aspectos que están aún por explorar. En la actualidad, la atención de los investigadores se centra en el desarrollo y cambio de actitudes de padres, profesores y alumnos, así como en las técnicas adecuadas para ello (p. ej., el role-playing, la cooperación a través del juego o de la interdependencia de aprendizajes u objetivos, etc.). Un primer paso necesario es, sin embargo, el reconocimiento y medición de las actitudes. El presente estudio trata de aportar algunas opciones viables para ello.

\section{Anexo}

\section{No 1. Escala de actitudes matemáticas del niño}

1. Las matemáticas me encantan y me gustan más que ninguna otra asignatura (AGR).

2. Disfruto viendo con qué rapidez y precisión puedo resolver problemas matemáticos (AGR).

3. Me gusta pensar ea cuestiones de matemáticas fuera de la escuela (AGR).

4. Algunas veces disfruto pensando en la forma de resolver problemas de matemáticas (AGR).

5. Nunca me aburro de trabajar con números (AGR).

6. Nunca me han gustado las matemáticas (TE).

7. Creo que las matemáticas son la asignatura más agradable de las que yo he estudiado (AGR).

8. Me gustaría dedicar más tiempo a cosas de matemáticas en la escuela (AGR).

9. Generalmente, odio las matemáticas y evito usarlas siempre que puedo (TE).

10. Me gustan las matemáticas porque son prácticas y útiles (UTI).

11. Nunca le he visto valor a las matemáticas (TE).

12. Siempre me han dado miedo las matemáticas (TE).

13. Me gustan las matemáticas tanto como las otras asignaturas (AGR).

14. Las matemáticas son muy interesantes (UTI). 
15. Las matemáticas me hacen sentir incómodo y nervioso (TE).

16. Siempre disfruto estudiando matemáticas en la escuela (AGR).

17. Las matemáticas son agradables y estimulantes para mí (AGR).

18. Nunca me han gustado las matemáticas y es la asignatura que más temo (TE).

19. Me gusta hacer más poblemas matemáticos de los que me mandan (AGR).

20. Me encanta utilizar las matemáticas fuera de la escuela (GR).

21. Las matemáticas me hacen sentir intranquilo y confuso (TE).

22. Las matemáticas son una materia necesaria y que merece la pena estudiar (UTI).

23. Todo el mundo debería estudiar matemáticas (UTI).

\section{N. 2. Escala de actitudes matemáticas de los padres}

1. Nunca me aburno de trabajar con números (AGR-UTI).

2. Generalmente, odio las matemáticas y evito usarlas siempre que puedo (TE).

3. Siempre me han dado miedo las matemáticas (TE).

4. Nunca me han gustado las matemáticas (TE).

5. Me gustan las matemáticas porque son prácticas y útiles (UTI).

6. Le temo a cualquier tipo de problema matemático (TE).

7. Las matemáticas son muy interesantes (AGR-UTI).

8. Nunca le he visto valor a las matemáticas (DES).

9. No hay nada creativo en las matemáticas; sólo es memorización de fórmulas y cosas (DES).

10. Las matemáticas son importantes para ayudar al progreso del mundo (AGR-UTI).

11. Los artistas y escritores deberían estudiar matemáticas igual que los científicos (AGR-UTI).

12. Las matemáticas son necesarias para, prácticamente, todas las cosas (AGR-UTI).

13. Las matemáticas son menos importantes para la gente que el arte y literatura (DES).

14. Todo el mundo debería estudiar matemáticas (AGR-UTI).

15. Las matemáticas son poco importantes en la vida diaria (DES).

16. Las matemáticas son una materia necesaria y que merece la pena estudiar (AGR-UTI).

17. Me gustan las matemáticas porque siempre hay una respuesta correcta (AGR-UTI).

18. Las matemáticas son agradables y estimulantes para mí (AGR-UTI).

19. Las matemáticas me hacen sentir incómodo y nervioso (TE).

20. Las matemáticas son pesadas y aburridas porque no dejan cabida para la creatividad ni la opinión personal (DES).

21. Intento adquirir nuevos conocimientos en matemáticas (AGR-UTI).

22. Nunca me han gustado las matemáticas y es la asignatura que más temí (TE).

23. Las matemáticas me hacen sentir intranquilo y confuso (TE).

\section{Nota}

Los ítems que forman las escalas de los padres y profesores son los mismos. La diferencia estriba en la ordenación de dichos ítems (azar), así como en ligeras variantes en cuanto a la redacción de éstos (verbos en pasado en el caso de los padres, por ejemplo).

\section{Referencias}

AIKEN, L. (1972). Biodata correlates of attitudes towards mathematics in three age and two sex groups. School Science and mathematics, 72, 386-395.

AIKEN, L. (1976). Update and attitudes and other affective variables in learning mathematics. Review of Educational Research, 46, 293-311.

Aiken, L., y DreGER, R. (1961). The effect of attitudes on performance in mathematics. Jr. of $E d u$ cational Psychology, 52, 19-24.

AlperT, et. al. (1963). Psychologycal factors in mathematics educations. Reports Summary in Newsletter $n^{\circ}$. 15. School Mathematics Study group. Stanford University.

BANKS, J. (1964). Learning and teaching arithmetic (2nd edition). Boston. Allyn y Bacon.

Berstein, B. (1964). Motivations in mathematics. School Science and Mathematics, 64, 749-754.

BurbanK, I. (1968). Relationships between parental attitudes toward mathematics and student attitude toward mathematics and students achievement in mathematics. Dissertation Abstracts International, 30, 3359A-3390A.

Do Carmo de Avila, M., y Guillet, L. (1970). Estude de l'attitude et de l'attitude envers les mathematiques. Bulletin de Psychologie Scholarie et d'Orientation, 19, 79-87.

Dutron, W. (1954). Measuring attitudes toward arithmetic. Elementary School Joumal, 55, 24-31.

Escamez, J., y OrTEGA, P. (1986). La enseñanza de actitudes y valores. Valencia. NAU llibres.

Fennema, E. (1980). Teacher and sex bias mathematics. Mathematics Teacher, 3, 169-173. 
Fennema, E., y Sherman, J. (1977). Sex-related differences in mathematics achievement spatial visualization and affective factors. American Educational Research Jr, 14, 51-57.

GaIRIN, J. (1987). Las actitudes en educación. Un estudio sobre educación matemática. Barcelona. PPU.

JACKSON, R. (1974). The attitudes of disadvantaged students toward mathematics. Dissertation Abstracts Intemational, 34, 3690A.

Kulharni, S., y NaIDU, C. (1970). Mathematics achievent realted student's socioeconomic and attitudes variables: A pilot study. Indian Joumal of Psychology, 45, 53-66.

Poffenberger, T., y Norton, D. (1959). Factors in the formation of attitudes toward mathematics. Joumal of Educational Research, 52, 171-176.

Quiles, M. (1986). La actitud y el rendimiento escolar en matemáticas: Un acercamiento multidimensional. Tesis doctoral. Universidad de La Laguna.

Quiles, M. (1988). Relación de las actitudes de padres y profesores con la actitud matemática del niño. Revista Portuguesa de Educaçao, 1, 103-110.

SAughnessy, J., et al. (1983). A causal analysis of attitudes toward mathematics. Jr. for Research in matbematics Education, 14, (1), 19-29.

SPICKERMAN, W. (1970). A study of the relationships between attitudes towards mathematics and some selected pupil characteristics in a Kentucky high school. Dissertation Abstracts International, 30, 2733AA.

WEBB, R. (1972). A study of the effects of anxiety and attitudes upon achievement in doctoral educational statistics courses. Dissertation Abstracts International, 32, 4997A-4998A.

\section{Actitudes matemáticas y rendimiento escolar. $M^{a}$. Nieves Quiles CL\&E, 1993, 18, pp. 115-125}

Resumen: Con el fin de comprobar si el rendimiento del alumno en matemáticas se ve afectado por las actitudes que hacia esa asignatura mantienen los padres, profesores y el propio alumno, llevamos a cabo un estudio correlacional. Participaron en él 600 alumnos de $50^{\circ}$ de EGB, 300 padres y 24 profesores. Las variables predictoras se midieron a través de tres escalas de actitudes matemáticas elaboradas para este estudio. La variable criterio, a su vez, se midió por la nota final obtenida en junio en dicha asignatura. Los resultados confirmaron la relación en el caso de padres y alumnos pero no en el de profesores.

Dirección: Departamento de Psicología Cognitiva, Social y Organizacional. Universidad de La Laguna. Tenerife. Islas Canarias.

Agradecimientos: A los profesores Armando Rodríguez y Bernardo Hernández por las correcciones y sugerencias a este artículo.

(C) De todos los artículos deberá solicitarse por escrito autorización de CL\&E y de los autores para el uso en forma de facsímil, fotocopia o cualquier otro medio de reproducción impresa. CL\&E se reserva el derecho de interponer las acciones legales necesarias en aquellos casos en que se contravenga la ley de derechos de autor. 